Special issue of the 2nd International Conference on Computational and Experimental Science and Engineering (ICCESEN 2015)

\title{
Analysis of Poly(2-Hydroxyethyl Methacrylate)-co- Poly(4-Vinyl Pyridine) Copolymers [COP2,4] Irradiated: an EPR study
}

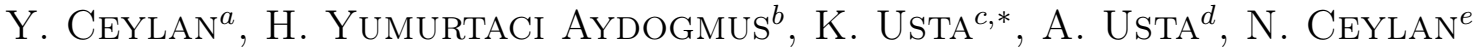 \\ AND H. YILMAZ ${ }^{f}$ \\ ${ }^{a}$ Selcuk University, Department of Physics, Konya, Turkey \\ ${ }^{b}$ Alanya Alaaddin Keykubat University, Department of Industrial Engineering, Antalya, Turkey \\ ${ }^{c}$ Alanya Alaaddin Keykubat University, Department of Metallurgical and Materials Engineering, Antalya, Turkey \\ ${ }^{d}$ Alanya Alaaddin Keykubat University, Department of Mechanical Engineering, Antalya, Turkey \\ ${ }^{e}$ Giresun University, Department of Chemistry, Giresun, Turkey \\ ${ }^{f}$ Gazi University, Department of Chemistry, Polatli-Ankara, Turkey
}

\begin{abstract}
Poly(2-hydroxyethyl methacrylate)-co-poly(4-vinyl pyridine) and poly(HEMA)-co-poly-(4-VP) copolymers were synthesized by free radical polymerization. $\mathrm{K}_{2} \mathrm{~S}_{2} \mathrm{O}_{8}$ was used as an initiator. Chain lengths of the copolymer was changed by varying the monomer/initiator ratio. These polymers have molarites of 2.6 and 2.1 respectively and are called COP2 and COP4. The samples were exposed to gamma rays at room temperature. After irradiation, the EPR spectra of COP2 and COP 4 were recorded between $120 \mathrm{~K}$ and $450 \mathrm{~K}$. From the temperature dependence of the line intensity, it was concluded that unpaired spin concentration in the irradiated samples has been changing with temperature. A theoretical study, presented in this report, was aimed to test success of the machine learning methods and to select the best learning method.
\end{abstract}

DOI: 10.12693/APhysPolA.130.167

PACS/topics: 87.66.Uv, 36.20.Kd, 39.30. + w, 33.35. + r

\section{Introduction}

Polymer structures, especially copolymers, are attractive due to their mechanical [1], optical [2-4] and electrical $[5,6]$ properties. Hydrophilically modified polymers have been used in coatings, paints, cosmetics, drilling fluids, in food industry, for petroleum recovery and also in the drug release systems in medical industry [7-9].

The irradiation has important role in polymer chemistry [10]. Generally polymerization and modification of the multicomponent polymer structures have been performed by radiation, because the reactions do not require initiators or catalysts. Sometimes irradiation results in bond scission, degradation of polymer structures [11] and eventually trapped radicals may occur [12]. EPR is a powerful spectroscopic method for detecting radicals in the materials [13-15].

In this study the EPR technique was used for detection of produced radical and measurement of their halflife time. It was also investigated whether the radical concentrations depend on temperature. The copolymers used in the study were synthesized by Hasim Yilmaz at the department of chemistry at Gazi University. The polymer samples used in the study have some interesting properties, for instance, when the powder copolymer in solvent is exposed to electric field, the powder structure

\footnotetext{
*corresponding author; e-mail: kezibanusta09@gmail.com
}

in the solvent changes into solid state, however in the absence of electric field, the solid structure again changes into viscous state.

\section{Experimental}

\subsection{Materials}

Purification of tetrahydrofuran (THF) (Aldrich, Germany) was performed in vacuum. HEMA (Aldrich, Germany) and 4-VP (Aldrich, Germany) were stored at temperatures below $273 \mathrm{~K}$. The monomers were purified by vacuum distillation before the monomers were transferred into the reaction vessel by a drop funnel under dry $\mathrm{N}_{2}(\mathrm{~g})$ atmosphere. Potassium persulfate $\left(\mathrm{K}_{2} \mathrm{~S}_{2} \mathrm{O}_{8}\right)$ (Aldrich, Germany) was used as initiator. The initiator was dried in vacuum oven at $343 \mathrm{~K}$ for at least two days.

\subsection{Polymerization}

HEMA, 4-VP, and $\mathrm{K}_{2} \mathrm{~S}_{2} \mathrm{O}_{8}$ were dissolved in THF at different compositions by keeping the mole number of one of the monomer constants. $1.0 \%$ of $\mathrm{K}_{2} \mathrm{~S}_{2} \mathrm{O}_{8}$ initiator was used in the polymerization mixture. All the glassware and transfer needles were dried by storing in oven at $413 \mathrm{~K}$ for overnight before the experiment of polymerization.

$\mathrm{K}_{2} \mathrm{~S}_{2} \mathrm{O}_{8}(0.1 \mathrm{~mol})$ was dissolved in $50 \mathrm{ml}$ of anhydrous THF at $60^{\circ} \mathrm{C}$ and then HEMA (six different amounts of $10,10,10,15,20$ and $25 \mathrm{ml}$ ) was added to the solution by dropwise method. The reaction mixture was stirred at $60^{\circ} \mathrm{C}$ for $2 \mathrm{~h}$. Subsequently 4 -VP (six different 
amounts of $15,20,25,10,10$ and $10 \mathrm{ml}$ ) was added to the prepared homo poly(HEMA) solution. This reaction proceeded at $333 \mathrm{~K}$ for about 32 hours. Afterwards, each of the viscous copolymer solutions was poured in a beaker. Cooled n-hexane was slowly added to this viscous solution to precipitate the copolymers separately. Polymerization was carried out in three-necked flask. All reactions were carried out under dry $\mathrm{N}_{2}(\mathrm{~g})$ atmosphere. Reaction mechanism of hydrolysis and formation of lithium salt of poly(HEMA)-co-poly(4-VP) copolymers is shown in Scheme 1, where $m, k$ is the mole number of used monomers and $m$ is identified by the following equation, $m=k+l$.<smiles>CCCCC(C)(CC(C)(C)C)C(=O)OCO</smiles>

In addition, the preparation conditions of the copolymers (COP2, COP4) are shown in Table I.

TABLE I

Preparation conditions of the copolymers.

\begin{tabular}{c|c|c|c|c|c}
\hline \hline Copolymer & Monomer-1 & Monomer-2 & $\begin{array}{c}\text { Volume-1 } \\
{[\mathrm{ml}]}\end{array}$ & $\begin{array}{c}\text { Volume-2 } \\
{[\mathrm{ml}]}\end{array}$ & $\begin{array}{c}{[1]+[2]} \\
{[\mathrm{mol} / \mathrm{l}]}\end{array}$ \\
\hline COP2 & HEMA & 4 -VP & 10 & 20 & 2.6 \\
COP4 & HEMA & 4 -VP & 15 & 10 & 2.1 \\
\hline
\end{tabular}

$[1]+[2] /\left[\mathrm{K}_{2} \mathrm{~S}_{2} \mathrm{O}_{8}\right]=100 ; T=(60.0 \pm 1.0)^{\circ} \mathrm{C} ; t=24 \mathrm{~h} ;$

solvent $=\mathrm{THF} ;$ non-solvent $=$ n-hexane

The precipitates were washed with methanol several times to remove residues of homopolymers and unreacted monomers. The resulting copolymers were dried in vacuum oven at $323 \mathrm{~K}$ for two days.

According to the synthesis order, the copolymers were coded as shown in Table I.

The copolymers with different compositions were produced by changing molar ratio of comonomers. The polymers with different molecular weight were obtained by varying the comonomer/initiator ratio.

Homo poly(HEMA) was polymerized at the beginning and then the second monomer, 4-VP, was added to the homo poly(HEMA) solution.

\subsection{EPR measurements}

The copolymer samples, called COP2 and COP4, were exposed to gamma source with dose rate of $0.980 \mathrm{kGy} / \mathrm{h}$ at room temperature for two days at Saraykoy Laboratory of Turkish Atomic Energy Authority. The polycrystalline samples of $0.8 \mathrm{~g}$ were placed into cylindrical EPR quartz tubes, and then the EPR measurements were performed at different temperatures between $120 \mathrm{~K}$ and $450 \mathrm{~K}$ using Bruker EMX 081 Spectrometer (X-Band, Germany). The settings of experimental system were as follows, microwave power of $5 \mathrm{~mW}$, modulation amplitude of $1 \mathrm{G}$, sweep width of $150 \mathrm{G}$.

\section{Theoretical}

\subsection{Machine learning method}

Machine learning is the common name of the computer algorithms that model a given problem according to the data from the environment of the problem. Machine learning is one of major branches of artificial intelligence which provides several indispensable tools for intelligent data analysis [16]. Artificial neural networks, decision trees, bayesian networks, support vector machines are examples of machine learning methods. Machine learning methods have been commonly used in many different areas for sampling and forecasting, and have high predictive accuracy. Recent successful applications of machine learning include stock market prediction [17], prediction of the future drainage chemistry [18], slump flow prediction [19], medical diagnosis [16] and face recognition [20] applications.

Machine learning algorithms consist of two phases; training phase and the testing phase. The dataset is divided into two parts for training and testing. Training set is used to train the system and a model is created in the first phase, then trained model generates estimates by using the test set.

\section{Results and discussion}

Formation of radicals in the copolymers (COP2 and COP4) irradiated with gamma rays was observed by EPR measurements. The measurements were performed in the range of 120-450 K. A single line was observed in the spectra of both copolymers. It was shown that the line intensity in the spectra was increasing in the range of 120-300 K, however above $300 \mathrm{~K}$, the intensity values were decreasing. We have observed the disappearance of
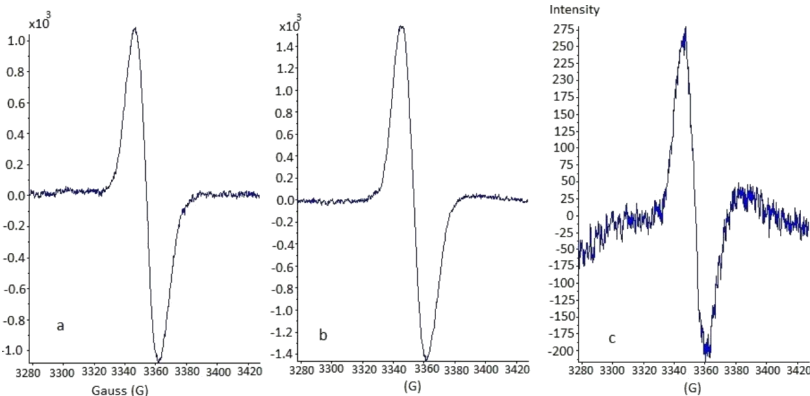

Fig. 1. EPR spectrum of radicals in COP4 at (a) $120 \mathrm{~K}$, (b) $290 \mathrm{~K}$ and (c) $390 \mathrm{~K}$.

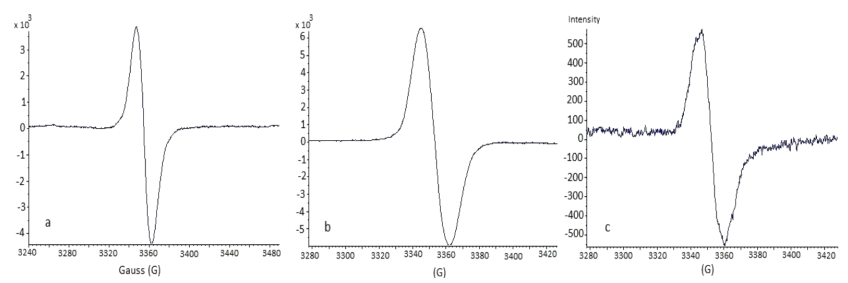

Fig. 2. EPR spectrum of radicals in $\mathrm{COP} 2$ at (a) $120 \mathrm{~K}$, (b) $290 \mathrm{~K}$ and (c) $430 \mathrm{~K}$. 
the signals above $390 \mathrm{~K}$ for COP 4 and above $430 \mathrm{~K}$ for COP2 (Figs. 1 and 2). From the line-shape and the linewidth in the spectra, it was concluded that the same radical structures were produced in the samples after gamma irradiation. Taking into account data from Table I and recorded spectra, it can be said that although COP2 has a smaller molarity than COP4, it has stronger bonds than COP4. in addition it was found that after irradiation, the color of COP2 had not changed, but the color of COP4 had.

Zainuddin et al. have studied gamma-irradiated poly(vinyl alcohol) [12]. They have observed four absorption lines in the recorded EPR spectra at $77 \mathrm{~K}$ and have assigned those to the presence of three radicals, an $\mathrm{C}_{\alpha}$-radical (a triplet), a superimposed neutral radical (a doublet), and an anion radical (a doublet). They have also found the absence of the doublet of neutral radicals and the occurrence of the triplet of the $\mathrm{C}_{\alpha}$-radical at increased temperature $(290 \mathrm{~K})$. Most polymers, exposed to gamma source, have changed mechanical properties from ductile to brittle. Suarez et al. have studied the correlation of mechanical and chemical changes in gammairradiated ultra-high-molecular-weight polyethylene [1].
Their experimental results have shown crosslinking and changing of molecular structure, degradation of the mechanical properties and changes in the fracture mechanism from ductile to brittle. The results of our experiments show that following irradiation, the COP4 polymer structure has changed the fracture mechanism from ductile to brittle, however no changes were observed in COP2. The measurements were performed at the same experimental conditions. It was determined from these results that the EPR spectra of the COP2 are similar to the spectra of the COP4, but the $g$ parameters have different values, $g$-values are 2.0167 for COP4 and 2.0139 for COP2. From these it was concluded that magnetic environment of unpaired electrons in the copolymers (COP2 and COP4) is different. After EPR measurements, we put the samples in the EPR tubes and then the samples were kept in liquid nitrogen for five days. Afterwards, EPR measurements were carried out again and the spectra were recorded. Considering these spectra, it was understood that the materials had stable radicals. The EPR spectra for COP4 is shown in Fig. 3a and for COP2 in Fig. 3b.

TABLE II

Statistical values of the training phase with COP2 data.

\begin{tabular}{c|c|c|c|c|c}
\hline \hline Method & $\begin{array}{c}\text { Correlation } \\
\text { coefficient }\end{array}$ & $\begin{array}{c}\text { Mean absolute } \\
\text { error }\end{array}$ & $\begin{array}{c}\text { Root mean } \\
\text { squared error }\end{array}$ & $\begin{array}{c}\text { Relative } \\
\text { absolute error, [\%] }]\end{array}$ & $\begin{array}{c}\text { Root relative } \\
\text { squared error, [\%] }\end{array}$ \\
\hline LAZY IBk & 0.9998 & 19.1031 & 36.0116 & 2.39 & 2.17 \\
Bagging RepTREE & 0.9989 & 46.6860 & 76.7145 & 5.85 & 4.62 \\
REPTree & 0.9982 & 58.3557 & 100.7975 & 7.31 & 6.07 \\
M5P & 0.9947 & 100.2402 & 173.7900 & 12.56 & 10.47 \\
M5Rules & 0.9930 & 113.2561 & 200.4699 & 14.00 & 12.00 \\
Regression by discretization & 0.9881 & 169.1283 & 255.1795 & 21.19 & 15.38
\end{tabular}

TABLE III

Statistical values of the training phase with COP4 data.

\begin{tabular}{c|c|c|c|c|c}
\hline \hline Method & $\begin{array}{c}\text { Correlation } \\
\text { coefficient }\end{array}$ & $\begin{array}{c}\text { Mean absolute } \\
\text { error }\end{array}$ & $\begin{array}{c}\text { Root mean } \\
\text { squared error }\end{array}$ & $\begin{array}{c}\text { Relative } \\
\text { absolute error, [\%] }\end{array}$ & $\begin{array}{c}\text { Root relative } \\
\text { squared error, [\%] }\end{array}$ \\
\hline LAZY IBk & 0.9996 & 8.0207 & 11.4151 & 4.24 & 2.92 \\
Bagging RepTREE & 0.9993 & 11.0247 & 14.9227 & 5.82 & 3.81 \\
REPTree & 0.9986 & 13.8677 & 20.4842 & 7.33 & 5.24 \\
M5P & 0.9954 & 23.9465 & 38.2856 & 12.65 & 9.79 \\
M5Rules & 0.9924 & 29.6261 & 49.4169 & 15.65 & 12.63 \\
Regression by discretization & 0.9883 & 41.0134 & 59.7557 & 21.67 & 15.28
\end{tabular}

At the implementation of the theoretical phase, studies were performed separately for COP2 and COP4. Unlike in our previous work [21], trainings were performed separately for the data sets and average statistical values were calculated. The basic steps of the theoretical study are as follows: firstly the current data was used for training for ten-fold cross validation in the Weka Explorer and it was tried to determine the methods having the least error values. Statistical results for the top six methods are shown in Tables II and III for COP2 and COP4, respectively. 
TABLE IV

Average statistical values of the training and test phases for COP2 data.

\begin{tabular}{c|c|c|c|c|c|c|c|c}
\hline \hline & \multicolumn{2}{|c|}{$\begin{array}{c}\text { Mean absolute } \\
\text { error }\end{array}$} & \multicolumn{2}{c|}{$\begin{array}{c}\text { Root mean } \\
\text { squared error }\end{array}$} & \multicolumn{2}{c|}{$\begin{array}{c}\text { Relative } \\
\text { absolute error, [\%] }\end{array}$} & \multicolumn{2}{c}{$\begin{array}{c}\text { Root relative } \\
\text { squared error, [\%] }\end{array}$} \\
\hline & Training & Test & Training & Test & Training & Test & Training & Test \\
\hline LAZY IBk & 19.294 & 108.543 & 36.679 & 154.900 & 2.447 & 12.116 & 2.212 & 9.081 \\
Bagging RepTREE & 47.811 & 118.044 & 78.747 & 199.567 & 6.064 & 13.038 & 4.750 & 11.468 \\
REPTree & 59.072 & 127.778 & 102.475 & 214.179 & 7.492 & 14.141 & 6.180 & 12.353
\end{tabular}

TABLE V

Average statistical values of the training and test phases for COP4 data.

\begin{tabular}{c|c|c|c|c|c|c|c|c}
\hline \hline & \multicolumn{2}{|c|}{$\begin{array}{c}\text { Mean absolute } \\
\text { error }\end{array}$} & \multicolumn{2}{c|}{$\begin{array}{c}\text { Root mean } \\
\text { squared error }\end{array}$} & \multicolumn{2}{c|}{$\begin{array}{c}\text { Relative } \\
\text { absolute error, [\%] }\end{array}$} & \multicolumn{2}{c}{$\begin{array}{c}\text { Root relative } \\
\text { squared error, [\%] }\end{array}$} \\
\hline LAZY IBk & 8.191 & 24.875 & 12.028 & 34.711 & 4.124 & 12.028 & 2.790 & 8.810 \\
Bagging RepTREE & 12.049 & 33.459 & 17.067 & 51.214 & 5.980 & 16.347 & 3.897 & 13.190 \\
REPTree & 15.366 & 34.228 & 24.722 & 52.197 & 7.593 & 16.699 & 5.537 & 13.424
\end{tabular}

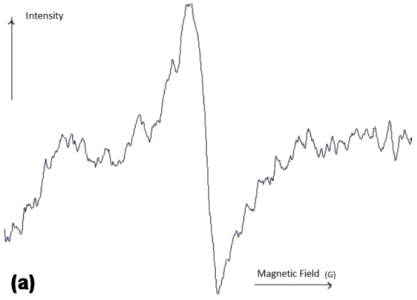

(b)

Fig. 3. Recorded EPR spectra of the COP4 (a) and COP2 (b).

The results obtained in this phase helped to determine the methods that will be used in subsequent training and testing phases. Secondly, training and test data sets were created for all temperatures, then training and testing phases were performed separately for the first three methods. The average statistical values of each data set, calculated in the training and test phases, are shown in Tables IV and V.

The results obtained in training and testing phases show that Lazy IBK gave the best results. Comparison of the predicted values, obtained by Lazy IBK, and actual values is shown in Figs. 4 and 5, for test phase.

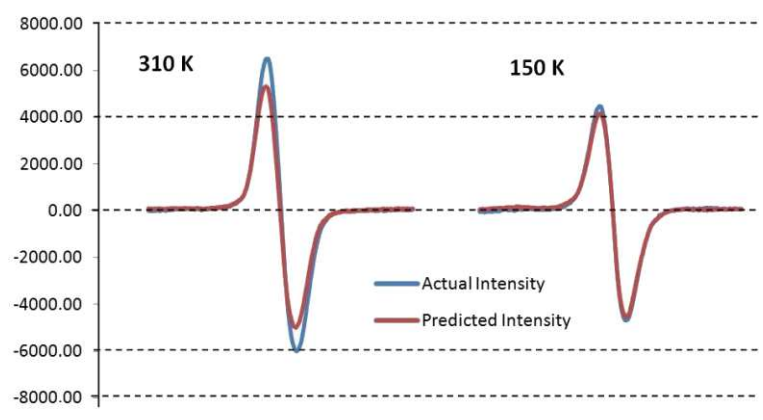

Fig. 4. Comparison of actual and predicted values for test phase of COP2 data (Lazy IBK method).

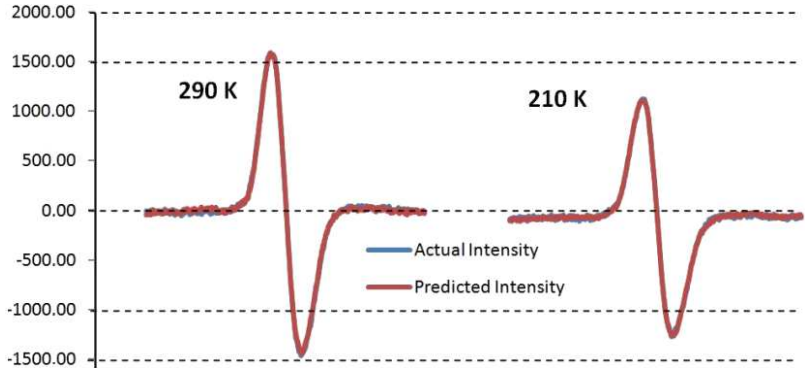

Fig. 5. Comparison of actual and predicted values for test phase of COP4 data (Lazy IBK method).

\section{Conclusions}

The results of our experiments have shown that after irradiation, fracture mechanism of the COP4 polymer was changed from ductile to brittle, however no change was observed in COP2. In addition, the color of COP4 polymer has changed and its color became claret, but the color of COP2 polymer did not change after the irradiation. The measurements were performed at the same experimental conditions. It was determined from these results that the EPR spectra of the COP2 are similar to the spectra of the $\mathrm{COP} 4$, but the $g$ parameters have different values. It was concluded from these results that magnetic environment of unpaired electrons in the copolymers (COP2 and COP4) is different.

At implementation of the theoretical phase, studies were performed separately for COP2 and COP4. The results obtained in training and testing phases have shown that Lazy IBK gave the best results.

\section{Acknowledgments}

This work is partially supported by the Alanya Alaaddin Keykubat University, BAP offices of Selcuk, and Gazi Universities. 


\section{References}

[1] J.C.M. Suarez, A. Elzubair, C.M.C. Bonelli, R.S. Biasi, E.B. Mano, J. Polym. Eng. 25, 277 (2005).

[2] W. Di, A. Petr, C. Kvarnstrom, L. Dunsch, A. Ivaska, J. Phys. Chem. C 111, 16571 (2007).

[3] D.R. McCamey, H.A. Seipel, S.Y. Paik, M.J. Walter, N.J. Borys, J.M. Lupton, C. Boehme, Nature Mater. 7, 723 (2008).

[4] I. Maher, H. Klaus Roth, M. Schroedner, A. Konkin, U. Zhokhavets, G. Gobsch, P. Scharff, S. Sensfuss, Organic Electronics 6, 65 (2005).

[5] T. Furukawa, H. Matsui, H. Hasegawa, S. Karuppuchamy, M. Yoshihara, Solid State Commun. 142, 99 (2007).

[6] A. El-Khodary, A.H. Oraby, M.M. Abdelnaby, J. Magnet. Magn. Mater. 320, 1739 (2008).

[7] M. Kuzuya, S. Kondo, Y. Sasai, Pure Appl. Chem. 77, 667 (2005).

[8] S. Biedermann-Brem, K. Grob, P. Fjeldal, Eur. Food Res. Technol. 227, 1053 (2008).

[9] P. Deo, P. Somasundaran, Langmuir 21, 3950 (2005).

[10] A.A. Zezin, V.I. Feldman, Radiat. Phys. Chem. 63, 75 (2002).
[11] D. Boxue, G. Yu, Polym. Degrad. Stabil. 94, 139 (2009).

[12] Zainuddin, D.J.T. Hill, T.T. Le, Radiat. Phys. Chem. 62, 283 (2001).

[13] E. Aras, B. Asik, M. Eken, M. Birey, Radiat. Eff. Defects S. 161, 373 (2006).

[14] M.A. Merdan, B. Asik, M. Birey, E. Aras, Radiat. Eff. Defects S. 167, 179 (2012).

[15] O. Karatas, E. Aras, J. Mol. Struct. 1027, 49 (2012).

[16] I. Kononenko, Artif. Intell. Med. 23, 89 (2001).

[17] R. Choudhry, K. Garg, WASET 39, 315 (2008).

[18] G.D. Betrie, R. Sadiq, K.A. Morin, S. Tesfamariam, Sci. Total Environ. 490, 182 (2014).

[19] H. Yumurtaci Aydogmus, H.I. Erdal, O. Karakurt, E. Namli, Y.S. Turkan, H. Erdal, Comput. Concrete 16, $741(2015)$.

[20] E. Osuna, R. Freund, F. Girosi, in: IEEE Computer Society Conference on Computer Vision and Pattern Recognition, 1997 p. 130.

[21] Y. Ceylan, K. Usta, A. Usta, H. Yumurtaci Aydogmus, A. Guner, J. Mol. Struct. 1100, 180 (2015). 